\title{
Special Issue of IJSR on Social Robots: Form, Content, Critique
}

\author{
Michaela Pfadenhauer • Satomi Sugiyama • \\ Charles M. Ess
}

Published online: 17 March 2015

(C) Springer Science+Business Media Dordrecht 2015

This special issue is an outcome of the COST Strategic Workshop on Social Robotic and Sustainability that took place in Brussels, Belgium, on 10-13 June 2013 (<http://www. cost.eu/events/socialrobotics $>$ ). We would like to begin by thanking both participants and organizers of the workshop for a very rich and inspiring event. Particular thanks go to Professor Leopoldina Fortunati (Facolta di Scienze della Formazione Universita degli Studi di Udine, Udine, Italy) who encouraged us to pursue the possibility of this special issue in conversation with the Editor in chief, Shuzhi S. Ge.

We have organized our issue around the three primary themes announced in the call, beginning with

(1) Form/appearance (e.g., human/animal likeness in appearance; everyday media forms and robotic functions; cultural attitudes toward robot forms, etc.)

In this section, we begin with Eleanor Sandry, "Reevaluating the form and communication of social robots: The benefits of collaborating with machinelike robots." Sandry highlights a first thematic for this special issue-namely, that among the manifold disciplines required for adequate attention to social robots, media and communication studies must be included for what these fields can offer in terms of better understanding what is required for human sociability

\footnotetext{
M. Pfadenhauer

Institut für Soziologie, Universität Wien, Vienna, Austria

e-mail: michaela.pfadenhauer@univie.ac.at

S. Sugiyama

Department of Communication and Media Studies, Franklin

University, Sorengo-Lugano, Switzerland

e-mail: ssugiyama@fus.edu

C. M. Ess $(\bowtie)$

Department of Media and Communication, University

of Oslo, Oslo, Norway

e-mail: c.m.ess@media.uio.no
}

in the broadest sense, i.e., as a (mediated) communicative engagement.

Equally importantly, Sandry announces a core normative concern shared by many in social robotics-namely, that fully human-like sociability includes a fundamental respect for the other as Other, i.e., as an entity to whom we owe the entire range of (originally human) set of rights and respect, including, to paraphrase Levinas (Sandry's primary philosophical source), a right to remain different. Broadly, our theories of sociability must includes these normative coresand in particular, as Sandry puts it, they must foster "a sense of the otherness of the machine and respect for its non-human abilities."

The subsequent two papers nicely complement Sandry's beginnings. So the contribution by Jakub Zlotowski, Diane Proudfoot, Kumar Yogeeswaran, and Christoph Bartneck takes up anthropomorphism from both philosophical perspectives and empirical findings from Human-Robotic Interaction and social psychology. The shared goal here is to better understand how anthropomorphism as a set of functions and processes can thereby guide us in the further development of social robots.

Lastly, especially the cultural dimensions of our approaches to social robots are elucidated by Timo Kaerlein in his "The Social Robot as Fetish? Conceptual Affordances and Risks of Neo-Animistic Theory." Animism is widely discussed as a particular feature of Japanese culture that putatively correlates with greater comfort with humanoid robots - but one that, as Kaerlein argues, runs the risk of having us attend more to the technical object as a fetish and thereby ignoring the "power relations and normative settings working in the background." In this way, Kaerlein complements and reinforces especially Sandry's emphasis on our always attending to the (potential) Otherness of social robots. 
Our second category, Content/AI/applications, refers to applications that shape human-robot interactions. Here, the work of Rodolphe J. Gentili, Hyuk Oh, Di-Wei Huang, Garrett E. Katz, Ross H. Miller, and James A. Reggia on neural architectures is oriented precisely towards the communicative interest in developing robotic systems whose imitation of "dexterous reaching, pointing, and grasping" can thereby contribute to more human-like social interaction. The particular approach to doing so outlined here progresses beyond the current states of the art in ways that may thereby further enhance these dimensions of human-machine sociability.

Our third category focuses on Critical issues that undergird the above two categories, including ethics, intimacy, emotions, and authenticity. To begin with, Michaela Pfadenhauer and Christoph Dukat's empirical analysis of how the well-known robot Paro actually functions in a care-giving context resonates with the contributions in the Form/Content category as they attend to how the appearance of Paro, as intended, contributes to its effectiveness in care-giving. In particular, as they put it, in one variant of the care-giving structures to emerge, a professional care-taker's skillful use of Paro can unlock the "heart doors of memory" otherwise occluded by dementia. At the same time, however, Pfadenhauer and Dukat foreground important questions of how the use of robots such as Paro may alter extant roles, status, and thereby power-and this in ways that go beyond the simple "robots will replace humans" trope.

Finally, Nello Barile and Satomi Sugiyama remind us of the fruitfulness of taking up matters of social robotics through the lenses of media and communication studies: this lens foregrounds the mobile phone as a form of social robot insofar as we focus especially on the communicative dimensions of sociability. Barile and Sugiyama specifically attend to "the algorithmization of taste"-i.e., the ways in which algorithmically-based recommender systems (re)shape our aesthetic tastes and preferences. In this way, as they put it, we begin to carry "some traces of robots." This returns us to the sort of normative concerns announced in the opening article by Sandry, in which the insistence is on learning to respect the Otherness of social robots as they develop in sophistication-including relative degrees of autonomy as the foundational characteristic for respect and rights. Conversely, Barile and Sugiyama raise the concern with how our interactions with such recommender systems may thereby restrict our own autonomy, in however subtle but thereby more problematic fashion.

We close with great gratitude to our contributors and the many reviewers whose critical insight and acumen helped improve first versions of these chapters. 